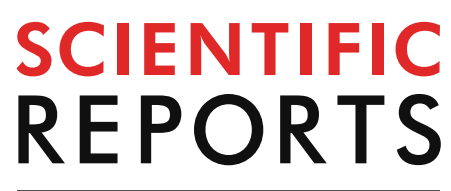

natureresearch

Check for updates

\title{
Parvalbumin-, substance P- and calcitonin gene-related peptide-immunopositive axons in the human dental pulp differ in their distribution of varicosities
}

\author{
Sook Kyung Park ${ }^{1,3}$, Seung Ki Choi ${ }^{1,3}$, Youn Gyung Kim ${ }^{1}$, So Young Choi ${ }^{2}$, Jin Wook Kim², \\ Sang Hyeok Seo ${ }^{1}$, Ji Hyun Lee ${ }^{1}$ \& Yong Chul Bae ${ }^{1 凶}$
}

Information on the frequency and spatial distribution of axonal varicosities associated with release of neurotransmitters in the dental pulp is important to help elucidate the peripheral mechanisms of dental pain, mediated by myelinated versus unmyelinated fibers. For this, we investigated the distribution of axonal varicosities in the human dental pulp using light- and electron-microscopic immunohistochemistry for the vesicular glutamate transporter 2 (VGLUT2), which is involved in the glutamatergic transmission, and syntaxin-1 and synaptosomal nerve-associated protein 25 (SNAP-25), combined with parvalbumin (PV), which is expressed mostly in myelinated axons, and substance $P(S P)$ and calcitonin gene-related peptide (CGRP), which are expressed mostly in unmyelinated axons. We found that the varicosities of the SP- and CGRP-immunopositive (+) axons were uniformly distributed throughout the dental pulp, whereas those of PV+ axons were only dense in the peripheral pulp, and that the expression of PV, VGLUT2, syntaxin-1, SNAP-25, SP and CGRP was significantly higher in the varicosities than in the axonal segments between them. These findings are consistent with the release of glutamate and neuropeptides by axonal varicosities of SP+ and CGRP+ unmyelinated fibers, involved in pulpal pain throughout the human dental pulp, and by varicosities of PV+ fibers, arising from parent myelinated fibers, and involved in dentin sensitivity primarily in the peripheral pulp.

The dental pulp is densely innervated by nociceptive neurons; the release of glutamate by their axons is a crucial part of the mechanism of signaling of acute nociceptive pain and of pathological pain ${ }^{1-4}$. The large majority of sensory neurons in the trigeminal ganglion (TG) that innervate the dental pulp give rise to myelinated (A) fibers and a few give rise to unmyelinated (C) fibers ${ }^{5}$. While the myelinated fibers are involved in the signaling of sharp, pricking, well-localized pain originating from the dentin and the peripheral pulp, the unmyelinated fibers are involved in signaling of dull, achy, poorly-localized pain originating from the pulp itself; the former play an important role in the dentin sensitivity, the latter play an important role in pulpal inflammatory pain ${ }^{6,7}$.

The pulpal axons frequently exhibit multiple varicosities along their length ${ }^{8-11}$, short segments where the axon is enlarged and contains multiple vesicles and mitochondria ${ }^{12,13}$. They also express two classes of proteins, which are involved in the loading of glutamate into synaptic vesicles and its release, the vesicular glutamate transporters (VGLUT) and the soluble $N$-ethylmaleimide sensitive factor attachment protein receptors (SNARE) ${ }^{9,10,14-16}$. These findings suggest that pulpal axons release glutamate from their varicosities. If this hypothesis is correct, it becomes important to collect information on the frequency and spatial distribution of varicosities of pulpal

${ }^{1}$ Department of Anatomy and Neurobiology, School of Dentistry, Kyungpook National University, 188-1, 2-Ga, Samdeok-Dong, Jung-Gu, Daegu 41940, Korea. ${ }^{2}$ Department of Oral and Maxillofacial Surgery, School of Dentistry, Kyungpook National University, Daegu 41940, Korea. ${ }^{3}$ These authors contributed equally: Sook Kyung Park and Seung Ki Choi. ${ }^{\bowtie}$ email: ycbae@knu.ac.kr 

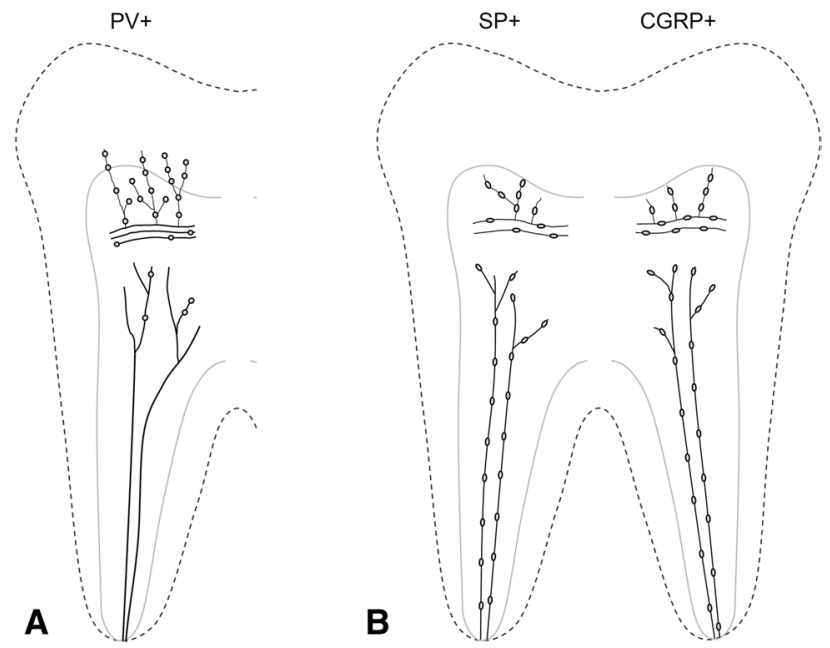

Figure 1. Diagrams showing the distribution of $\mathrm{PV}+(\mathbf{A})$, and $\mathrm{SP}+$ and $\mathrm{CGRP}+(\mathbf{B})$ axons and their varicosities in the human dental pulp. (A) While most PV+ axons display a large number of varicosities in the peripheral pulp, few PV+ axons have any varicosities in the radicular pulp and the core of the coronal pulp. (B) Almost all $\mathrm{SP}+$ and CGRP+ axons possess varicosities uniformly throughout the dental pulp.

axons arising from parent myelinated axons and unmyelinated axons, and correlate that to what is known about their role in mediating nociception.

For this, we here investigated the distribution of varicosities in the human dental pulp from human premolar teeth using light microscopic immunohistochemistry for VGLUT, combined with parvalbumin (PV), which is expressed mostly in parent myelinated axons, and substance P (SP) and calcitonin gene-related peptide (CGRP), which are expressed mostly in unmyelinated axons, in the sensory root of the trigeminal ganglion ${ }^{17,18}$. We also used electron microscopic immunohistochemistry to examine the varicosities that expressed PV, SP, CGRP, VGLUT2, and the SNARE proteins syntaxin-1 and synaptosomal nerve-associated protein 25 (SNAP-25).

\section{Results}

Distribution of axonal varicosities within the dental pulp. Most axons in the dental pulp that were immunostained for PV, SP or CGRP were within axonal bundles in the radicular pulp and occasionally in the core of the coronal pulp. The PV-immunopositive $(\mathrm{PV}+)$ axons branched extensively out of these bundles and towards the dentin, whereas the SP+ and CGRP+ axons branched frequently in the peripheral pulp, distal to the parietal nerve plexus (Figs. 1, 2).

While only few PV+ axons in the radicular pulp and the core of the coronal pulp had any varicosities, virtually every PV+ axon in the peripheral pulp displayed multiple varicosities. The SP+ and CGRP+ axons, on the other hand, did not show such regional differences and had equally dense varicosities throughout the dental pulp (Figs. 1,2). Quantitative analysis revealed that the number of varicosities per unit length of the PV+ axons was significantly higher in the peripheral pulp than in the core of the coronal and the radicular pulp. Conversely, for the SP+ and CGRP+ axons, it was similar among the three pulpal regions. The number of varicosities per unit length for $\mathrm{PV}+, \mathrm{SP}+$ and CGRP+ axons was similar in the peripheral pulp, whereas it was significantly higher for the SP+ and the CGRP+ axons than for the PV+ axons in the core of the coronal and radicular pulp (Table 1). The VGLUT2+, syntaxin-1+ and SNAP-25+ axons had numerous branches and varicosities in the peripheral pulp (Fig. 3). In the core of the coronal and the radicular pulp, many VGLUT2+ axons but few syntaxin-1+ or SNAP$25+$ axons had varicosities. In addition, double immunofluorescent staining revealed that the majority of SP+ and CGRP+ axons and few of the PV+ axons expressed VGLUT2 (Fig. 4). The fraction of VGLUT2-expressing $\mathrm{SP}+(32.6 \pm 7.8 \%)$ and CGRP+ $(39.2 \pm 5.9 \%)$ axons was significantly higher than that of VGLUT2-expressing $\mathrm{PV}+$ axons $(6.2 \pm 3.6 \%)$. PV+ axons did not co-stain for SP or CGRP, suggesting that in the dental pulp, PV is expressed in a different axon type than CGRP or SP (Fig. 5).

Ultrastructure of the axonal varicosities in the dental pulp. At electron microscopy, axonal varicosities were observed on unmyelinated fibers and on unmyelinated segments of myelinated fibers: Myelinated fibers entered the dental pulp as myelinated and then lost their myelin. The varicosities but not the axonal segments between them contained numerous small vesicles and mitochondria; the varicosities of SP+ and CGRP+ axons also contained large vesicles with dense cores. Varicosities were not covered by Schwann cells, which can be favorable structure for the release of neurotransmitters. The density of immunostaining for PV, SP, CGRP, VGLUT2, syntaxin-1 and SNAP-25 was significantly higher in the varicosities than in the interim segments of the axon, pointing to varicosities as a likely site of accumulation and release of glutamate and neuropeptides in the dental pulp (Fig. 6). 

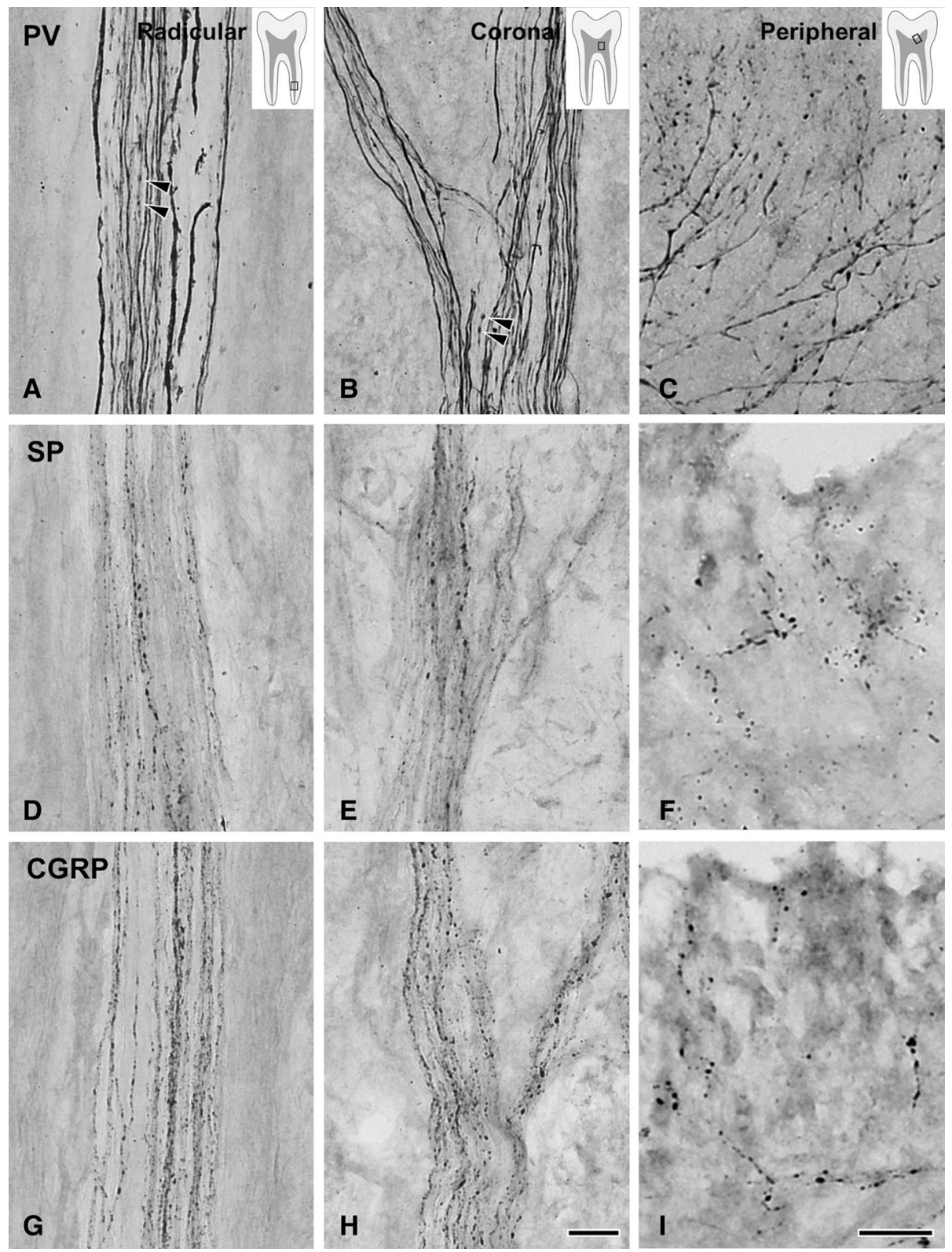

Figure 2. Light micrographs of PV+ (A-C), $\mathrm{PP}+(\mathbf{D}-\mathbf{F})$, and CGRP+ $(\mathbf{G}-\mathbf{I})$ axons in the radicular $(\mathbf{A}, \mathbf{D}$, $\mathbf{G})$, the core of the coronal $(\mathbf{B}, \mathbf{E}, \mathbf{H})$ and the peripheral $(\mathbf{C}, \mathbf{F}, \mathbf{I})$ regions of the human dental pulp. Most $\mathrm{PV}+$ axons have a large number of varicosities in the peripheral pulp $(\mathbf{C})$, whereas few $\mathrm{PV}+$ axons show occasional varicosities in the radicular pulp and the core of the coronal pulp (A, B). In contrast, almost all SP+ and CGRP+ axons possess varicosities throughout the dental pulp without any obvious regional differences (D-I). Arrowheads in (A, B) indicate PV+ axon varicosities in the radicular and the core of the coronal pulp. Rectangles in the insets in $(\mathbf{A}),(\mathbf{B})$, and $(\mathbf{C})$ indicate the pulp regions represented in the respective figures. Scale bar $=20 \mu \mathrm{m}$ (scale bar in $\mathbf{H}$ applies to A, B, D, E, G and in I applies to $\mathbf{C}, \mathbf{F}$ ).

\begin{tabular}{|c|c|c|c|}
\hline Pulpal region & $\mathbf{P V}^{1}$ & SP & CGRP \\
\hline Peripheral pulp & $14.72 \pm 2.61$ & $13.66 \pm 2.43$ & $14.22 \pm 0.54$ \\
\hline Coronal pulp ${ }^{2}$ & $1.55 \pm 0.25$ & $13.02 \pm 1.06$ & $13.78 \pm 0.76$ \\
\hline Root pulp ${ }^{2}$ & $0.82 \pm 0.33$ & $11.58 \pm 3.92$ & $12.41 \pm 0.96$ \\
\hline
\end{tabular}

Table 1. Number (Mean \pm SD from three pulps) of varicosities per $100 \mu \mathrm{m}$ length of axon in 3 regions of the human pulp. ${ }^{1}$ Significant difference between peripheral pulp and coronal/root pulp at the 0.01 level (one-way ANOVA, Scheffe's test). ${ }^{2}$ Significant difference between PV and SP/CGRP at the 0.01 level (one-way ANOVA, Scheffe's test). 

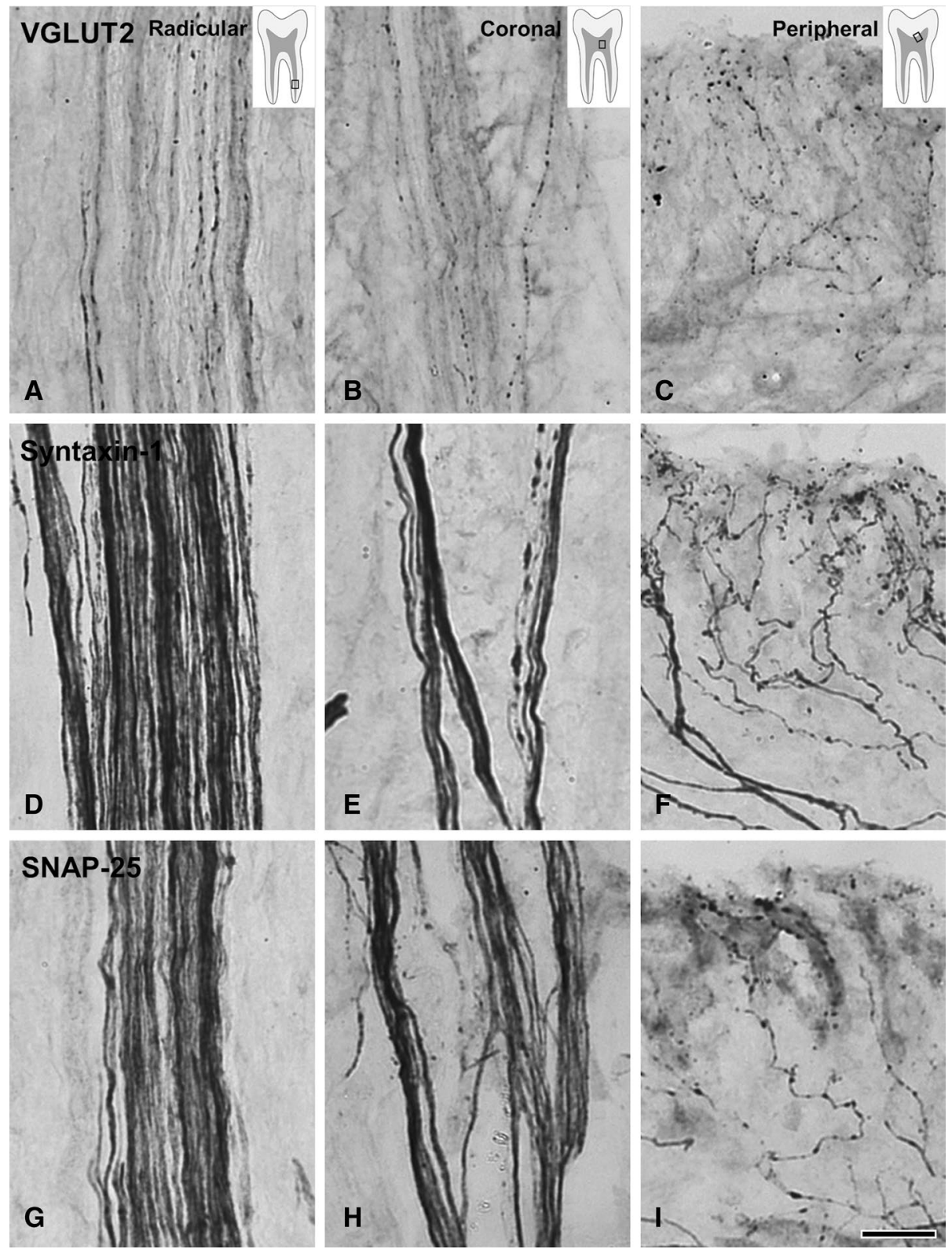

Figure 3. Light micrographs of VGLUT2+ (A-C), syntaxin-1+ (D-F), and SNAP-25+ (G-I) axons in the radicular $(\mathbf{A}, \mathbf{D}, \mathbf{G})$, the core of the coronal $(\mathbf{B}, \mathbf{E}, \mathbf{H})$, and the peripheral $(\mathbf{C}, \mathbf{F}, \mathbf{I})$ regions of the human dental pulp. Most of the VGLUT2+, syntaxin-1+, and SNAP-25+ axons show numerous varicosities in the peripheral pulp. Many VGLUT2+ axons and few syntaxin-1+ and SNAP-25+ axons show varicosities in the core of the coronal and in the radicular pulp. Electron-dense immunolabeling for VGLUT2, syntaxin-1, and SNAP-25 in the axonal stands and bundles is assumed to represent the proteins that are being transported from the neuronal cell bodies to the axonal varicosities. VGLUT2+ axons show weak immunostaining (A-C), in contrast to the dense immunostaining for syntaxin-1+ and SNAP-25+ in axonal strands and bundles (D-I). Scale bar $20 \mu \mathrm{m}$ (which applies to $\mathbf{A}-\mathbf{H}$ ).

\section{Discussion}

The main findings of the present study are that while the varicosities of the SP+ and CGRP+ axons were uniformly distributed throughout the human dental pulp, those of PV+ axons were only dense in the peripheral pulp, and that the expression of SP, CGRP, VGLUT2, syntaxin-1 and SNAP-25 was significantly higher in the varicosities than in the axonal segments between them. These observations support the hypothesis that axonal varicosities are sites of release of glutamate and neuropeptides by SP+ and CGRP+ unmyelinated fibers, involved in pulpal pain throughout the human dental pulp, and PV+ fibers, arising from parent myelinated fibers and involved in dentin sensitivity in the peripheral pulp only.

The axonal varicosities in the dental pulp contained vesicles and mitochondria and densely expressed VGLUT2, syntaxin-1 and SNAP-25, which are involved in vesicular loading and exocytosis of neurotransmitters 

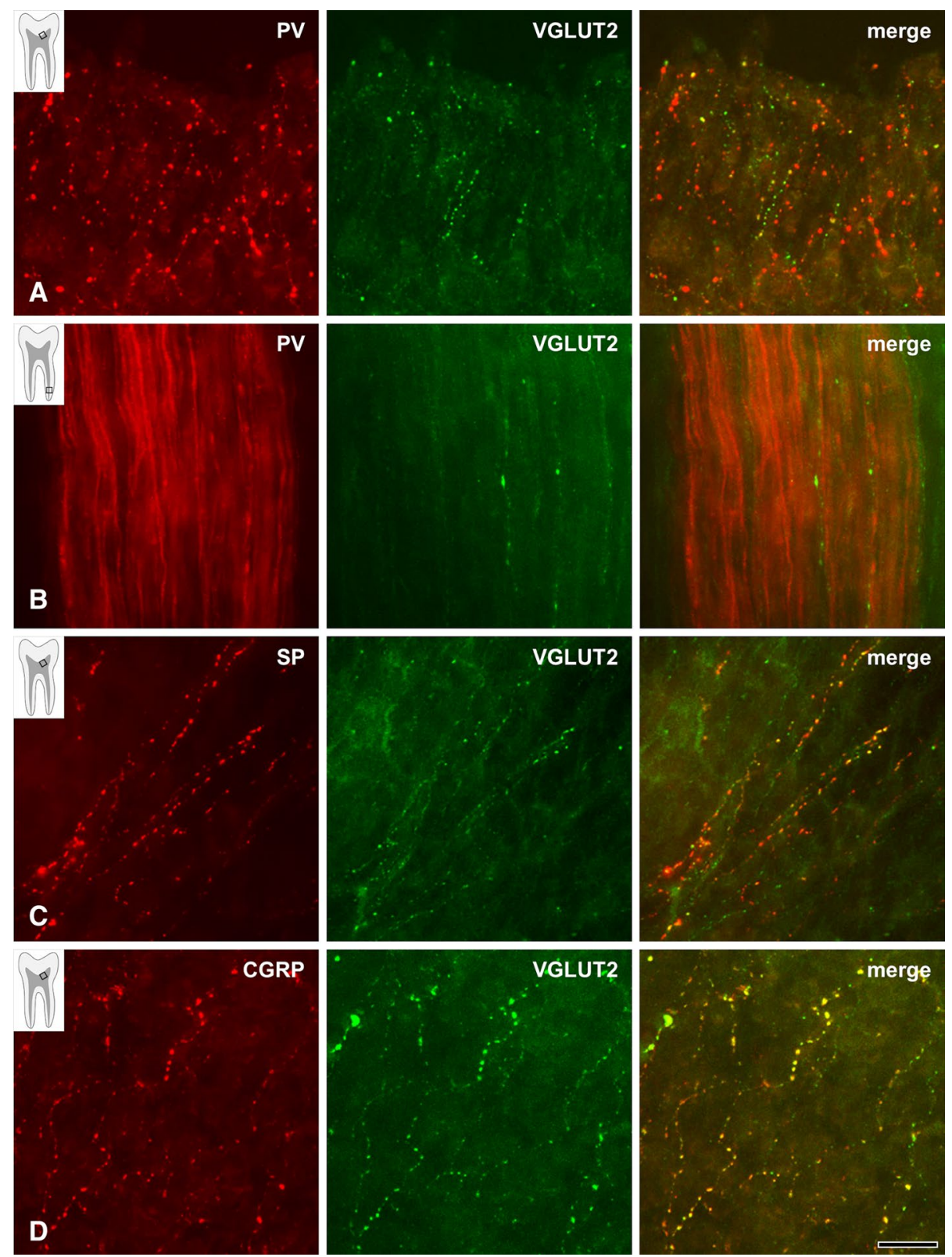

Figure 4. Double immunofluorescent staining for PV (red) and VGLUT2 (green) in the peripheral (A) and radicular (B) pulp, and for SP or CGRP (red) and VGLUT2 (green) in the peripheral pulp (C, D). Few PV+ axons (A) and the majority of SP+ (C) and CGRP+ (D) axons co-stain for VGLUT2, especially densely at their varicosities in the peripheral pulp. The PV+ axons rarely show varicosities and rarely co-stain for VGLUT2 in the radicular pulp (B). Insets indicate the pulp regions represented in the respective photomicrographs. Co-stain is shown in yellow in the merged images. Scale bar $20 \mu \mathrm{m}$.

and the neuropeptides SP and CGRP, which in turn, have been shown to mediate nociception and neurogenic inflammation ${ }^{2-4,19}$. This suggests that the axonal varicosities represent the site of release of glutamate and neuropeptides in the dental pulp, analogous to the varicosities of autonomic nerves, which also contain numerous vesicles and mitochondria, express SNARE proteins, and do not establish synaptic contact with adjacent effector cells $^{20,21}$.

Several lines of evidence suggest that the glutamate signaling by peripheral sensory axons plays a crucial role in acute nociception and pathological pain ${ }^{2-4,22}$. Thus, direct application of glutamate to peripheral tissues induces excitation of nociceptive $\mathrm{A} \delta$ and $\mathrm{C}$ fibers and their subsequent sensitization ${ }^{3}$, and injection of glutamate into deep facial tissues evokes acute pain and induces mechanical allodynia and heat hyperalgesia ${ }^{4,23,24}$. In addition, it has been shown that the pulpal axons express the metabotropic glutamate receptor mGluR5 and that its expression is increased following pulpal inflammation ${ }^{8,25}$. By analogy, acute and pathologic dental pain may be mediated by glutamate released from the varicosities of the pulpal axons. 

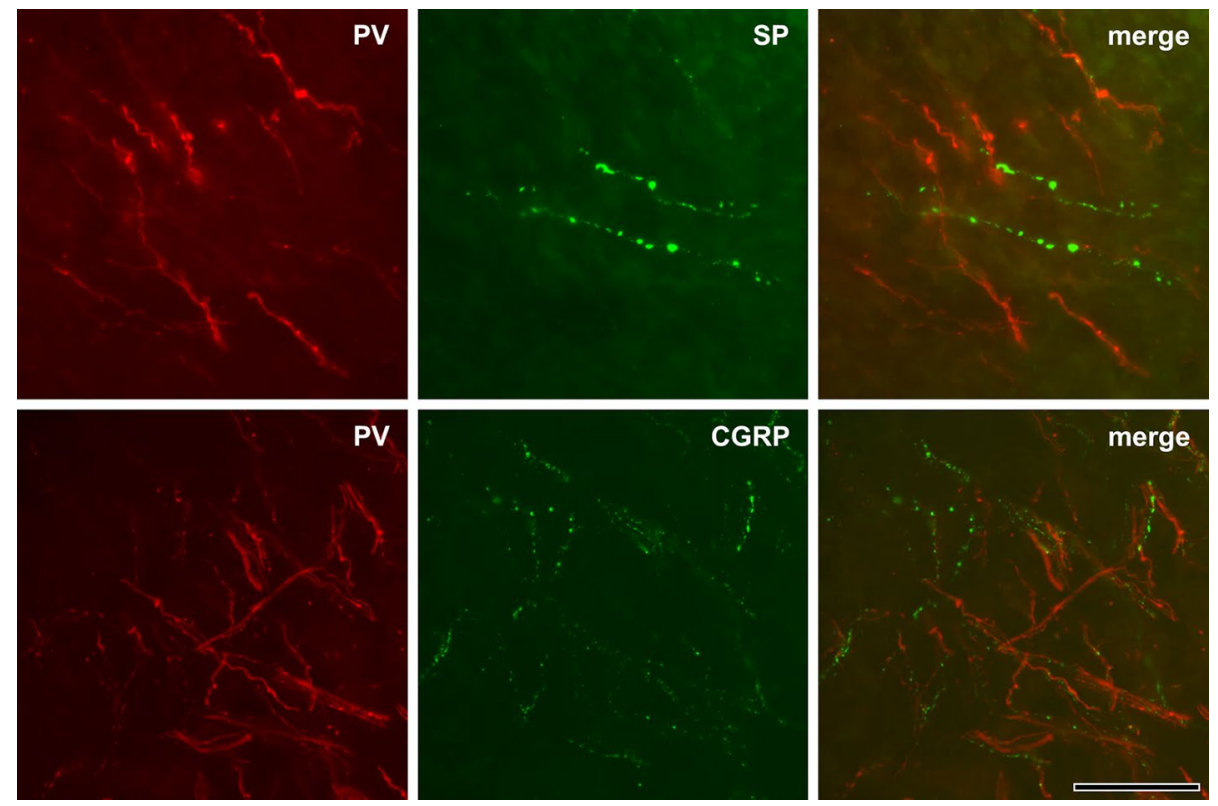

Figure 5. Double immunofluorescent staining for PV (red) and SP or CGRP (green) in the coronal region of the human dental pulp. PV+ axons do not co-stain for SP or CGRP. Scale bar $50 \mu \mathrm{m}$.

That the majority of SP+ and CGRP+ axons, but few of the PV+ axons coexpressed VGLUT2, which is analogous to the respective neurons in the $\mathrm{TG}^{26}$, suggests that the VGLUT2-dependent glutamate release in the dental pulp is mediated primarily by SP+ and CGRP+ unmyelinated axons, and to a much lesser degree by PV+ axons, arising from parent myelinated axons. Considering that the VGLUT1+ primary sensory neurons express mostly PV, NF200, and SSEA4, which are expressed in neurons with myelinated axons, and rarely CGRP and IB4, which are expressed in neurons with unmyelinated axons ${ }^{26-28}$, the pupal PV+ axons arising from parent myelinated axons are likely to express VGLUT1 and be involved in the VGLUT1-dependent glutamate release.

Considering that in the sensory root of TG $98.8 \%$ of the PV+ parent axons are myelinated, and $99.5 \%$ of the $\mathrm{SP}+$ and $96.5 \%$ of the CGRP+ parent axons are unmyelinated ${ }^{17,18}, \mathrm{PV}+$ fibers may arise from parent myelinated A fibers, and $\mathrm{SP}+$ and CGRP+ fibers may arise from unmyelinated C fibers. Since it is typical for myelinated fibers to lose their myelin sheath and become thinner during their course to the peripheral target ${ }^{29}$, PV+ fibers lose their myelin during their course to the dental pulp. Thus, $66 \%, 79 \%$ and $99 \%$ of the PV+ parent fibers, which are myelinated in the sensory root of the TG, become unmyelinated at the root pulp, coronal pulp and peripheral pulp, respectively ${ }^{30}$. Varicosities were observed within the unmyelinated segment of the PV+ fibers, but not within the their myelinated segment.

$\mathrm{PV}$ is expressed by a small subset of proprioceptive neurons in the dorsal root ganglion (DRG) ${ }^{18,31,32}$, but in a large number of low-threshold mechanoreceptive and nociceptive neurons in the $\mathrm{TG}^{18,33}$. Also, the human dental pulp, which has very few, if any, proprioceptors ${ }^{34-36}$, is densely innervated by a large number of PV+ axons ${ }^{30}$. Considering these findings, PV may label large number of pulpal axons arising from parent myelinated axons. SP and CGRP have been routinely used as markers for primary sensory neurons with unmyelinated fibers ${ }^{37}$ even though they have been shown to also label some DRG neurons with myelinated fibers ${ }^{38,39}$. That PV+ pulpal axons did not co-stain for SP or CGRP, which is consistent with findings in the rat dental pulp ${ }^{40}$, suggests that PV and SP/CGRP are expressed by distinct axon types.

In agreement with our previous observations, the PV+ axons branched extensively in the peripheral pulp distal to the parietal plexus of nerves, and many of them extended towards the dentinal tubules ${ }^{30}$. That almost all $\mathrm{PV}+$ axons possessed numerous varicosities in the peripheral pulp but few had any varicosities in the radicular pulp and the core of the coronal pulp suggests that glutamate released from numerous varicosities of PV+ axons, following excitation in the dentin and peripheral pulp, can induce dental hypersensitivity by activating adjacent and nearby axons. This possibility is supported by the observation that numerous axonal varicosities in the peripheral pulp also express mGluR5 ${ }^{8}$. On the other hand, that there are few PV+ axonal varicosities in the radicular pulp and the core of the coronal pulp is consistent with the observation that a considerable fraction of PV+ axons in these regions of the dental pulp are myelinated and thus do not express functional glutamate receptors and are unlikely target for either autocrine or paracrine activation and/or sensitization by glutamate ${ }^{30}$.

The peptidergic (SP+ or CGRP +) neurons in the TG coexpress the markers for glutamatergic transmission VGLUT $^{26,28,41,42}$ and glutaminase ${ }^{43}$. We also showed previously that pulpal SP+ or CGRP+ axons frequently coexpress VGLUT2 ${ }^{9}$. Taken together, these findings suggest that pulpal peptidergic $\mathrm{C}$ fibers may release glutamate as well as neuropeptides. Also, since at variance with the PV+ varicosities, the SP+ and CGRP+ varicosities were uniformly distributed throughout the dental pulp, the co-release of glutamate and neuropeptides and the subsequent autologous activation of these fibers or of adjacent fibers during inflammation, is more likely to occur uniformly throughout the dental pulp and not be confined to the peripheral pulp. 

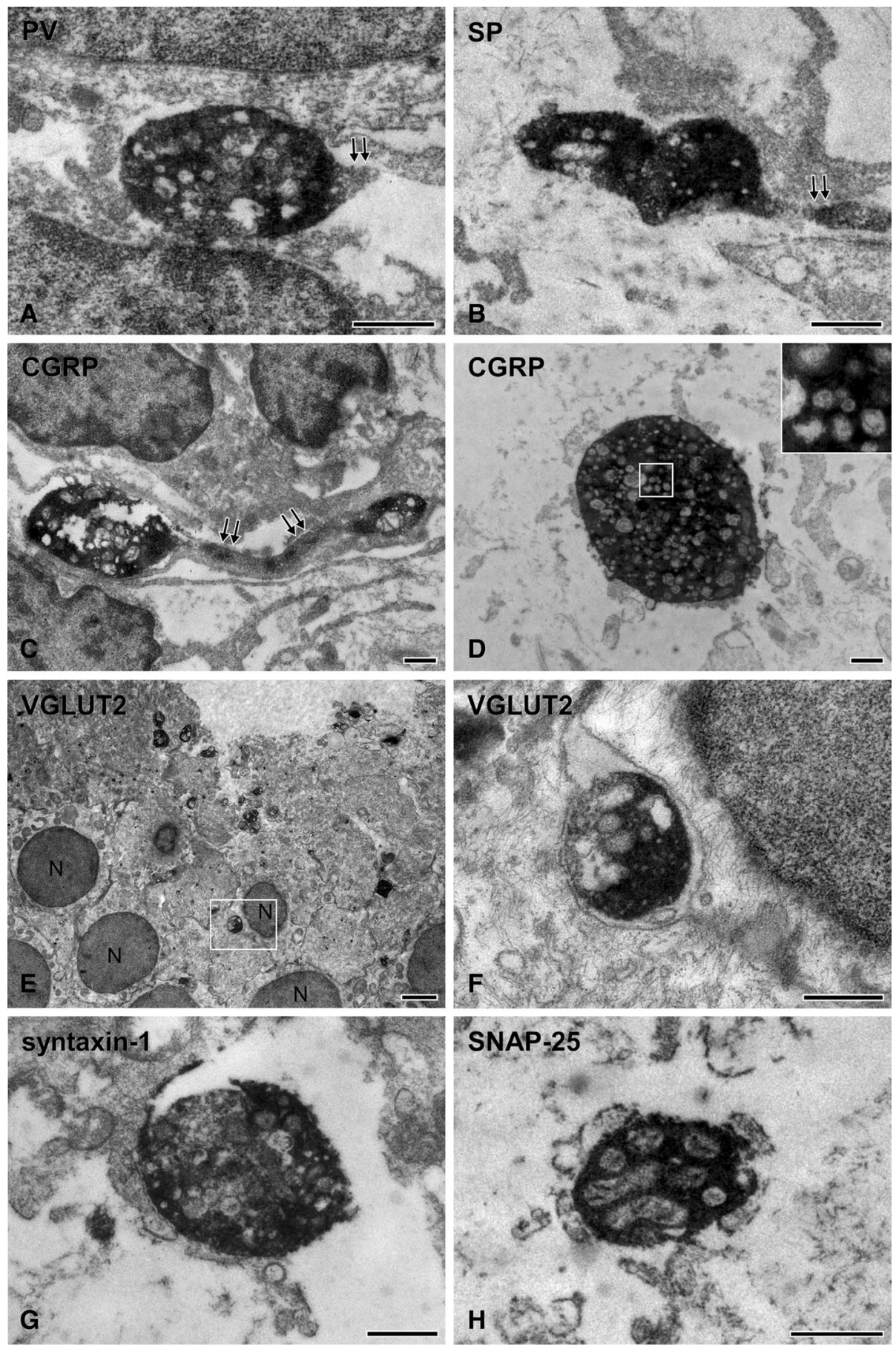

Figure 6. Electron micrographs showing PV+ (A), SP+ (B), CGRP+ (C, D), VGLUT2+ (E, F), syntaxin-1+ $(\mathbf{G})$, and SNAP-25+ (H) axonal varicosities in the human dental pulp. The immunoreaction product is dense in varicosities but weak and patchy in the axonal segments between them (double arrows in $\mathbf{A}-\mathbf{C}$ ). The varicosities contain numerous small vesicles and mitochondria. Note that CGRP+ varicosities also contain large vesicles with dense cores, thought to contain neuropeptides. The insets in (D)is an enlargement of the boxed area within the main image. (F) is enlargement of the boxed area in (E) and shows a VGLUT2+ varicosity contacting the cell body of an odontoblast. $\mathrm{N}$ indicates the nucleus of the odontoblast. Scale bar $500 \mathrm{~nm}$ in $(\mathbf{A}-\mathbf{D}),(\mathbf{F}-\mathbf{H})$ and $2 \mu \mathrm{m}$ in $(\mathbf{E})$.

\section{Methods}

We obtained the formal approval of the Research and Ethics Committee of the Kyungpook National University Dental Hospital prior to performing any experimental work. All experiments were carried out in accordance 
with the relevant guidelines and regulations. All human materials were collected for use after the subjects were explained the nature of the experiments and informed consent was obtained from all subjects or, if subjects are under 18, from a parent.

Tissue preparation. We used maxillary premolars from nine human subjects, 15-28 years of age; the teeth were healthy and were extracted for orthodontic indication at the Department of Oral Surgery, Kyungpook National University Hospital. The pulps were extracted in toto by cutting the teeth along their longitudinal axes. The tissues were fixed for $5 \mathrm{~h}$ in a solution of $4 \%$ paraformaldehyde in phosphate buffer $(\mathrm{PB}, 0.1 \mathrm{M}, \mathrm{pH} 7.4)$ for light microscopy ( 6 pulps) or in a mixture of $4 \%$ paraformaldehyde and $0.01 \%$ glutaraldehyde for electron microscopy ( 3 pulps). The tissues were then immersed in a solution of $30 \%$ sucrose in $\mathrm{PB}$ at $4{ }^{\circ} \mathrm{C}$. On the next day, 20-30 $\mu$ m-thick sections for light microscopy were cut on a cryotome, and 50-60 $\mu \mathrm{m}$-thick sections for electron microscopy were cut on a Vibratome (Leica Biosystems, Wetzlar, Germany).

Light microscopic immunohistochemistry. Two protocols were used to prepare sections for light microscopy, immunoperoxidase for single staining and immunofluorescence for double staining. For immunoperoxidase, the sections were incubated in PB-buffered solutions of $50 \%$ ethanol for $30 \mathrm{~min}, 3 \% \mathrm{H}_{2} \mathrm{O}_{2}$ for $10 \mathrm{~min}$, and 10\% normal donkey serum (NDS) (Jackson ImmunoResearch, West Grove, PA) for 10 min. They were then rinsed several times in $\mathrm{PB}$ and transferred to the primary antibody in phosphate-buffered saline (PBS; $0.01 \mathrm{M}, \mathrm{pH}, 7.4)$ for $18 \mathrm{~h}$. We used the following primary antibodies and dilutions: mouse anti-PV (235; Swant, Marly, Switzerland) at 1:3,000, rat anti-SP (MAB356; Millipore, Billerica, MA) at 1:500, mouse antiCGRP (ab81887; Abcam, Cambridge, MA) at 1:1,000, guinea pig anti-VGLUT2 (VGluT2-GP-Af670; Frontier Institute Co., Ltd, Hokkaido, Japan) at 1:500, mouse anti-syntaxin-1 (S0664; Sigma-Aldrich, St. Louis, MO) at 1:2,000 and rabbit anti-SNAP-25 (S9684; Sigma-Aldrich) at 1:3,000. On the next day, the sections were rinsed extensively in PBS and transferred to the appropriate secondary antibody, diluted to 1:200 in PBS for $2 \mathrm{~h}$. The following biotinylated secondary antibodies were used: donkey anti-mouse, donkey anti-rat, donkey anti-guinea pig and donkey anti-rabbit (all from Jackson ImmunoResearch). Avidin-biotin-peroxidase binding was with ExtrAvidin peroxidase (Sigma-Aldrich) at 1:5,000 for $1 \mathrm{~h}$. Finally, the peroxidase was revealed according to the nickel-intensified 3,3'-diaminobenzidine tetrahydrochloride (Ni-DAB) protocol. Immunostained sections were then coversliped on slides with Permount (Fisher).

For immunofluorescence, the sections were pretreated with ethanol and NDS as above and transferred to a mixture of guinea pig anti-VGLUT2 antibody (1:500) and rabbit anti-PV (1:2,000, PV 25, Swant, Marly, Switzerland), rabbit anti-SP $(1: 1,000,20,064$; immunostar; Hudson, WI) or mouse anti-CGRP antibody(1:1,000) or a mixture of rabbit anti-PV $(1: 2,000)$ and guinea pig anti-SP (1:1,000, AB5892, Chemicon, Temecula, CA) or mouse anti-CGRP $(1: 1,000)$ for an overnight incubation. After that, the sections were rinsed and incubated with a donkey anti-mouse or a donkey anti-rabbit antibody labeled with $\mathrm{Cy} 3$ in a mixture with a donkey anti-guinea pig or donkey anti-mouse antibody labeled with fluorescein isothiocyanate (Jackson ImmunoResearch) at 1:200 for $3 \mathrm{~h}$. Finally, the sections were rinsed extensively and mounted on slides with Vectashield (Vector). Slides were examined on a Zeiss Axioplan 2 microscope (Carl Zeiss Inc., Jena, Germany) and a confocal microscope (LSM 510 Meta; Carl Zeiss Inc.).

Quantitative analysis. The number of varicosities per unit axonal length was determined using sections stained with immunoperoxidase. Images were obtained at $40 \times$ with an Exi digital camera (Q-imaging Inc., Surrey, CA) attached to a Zeiss Axioplan 2 microscope (Carl Zeiss, Göttingen, Germany), and saved as TIFF files. The number of varicosities and the length of the PV+, SP+ and CGRP+ axons in each peripheral, coronal, and radicular pulp were measured from a total of 12-16 images from each pulpal region in 3-4 sections of each of three human dental pulps. Continuous strings of axonal beads with an apparent linear arrangement were considered a single axon.

The fractions of PV+, SP+ and CGRP+ axons that coexpress VGLUT2 were analyzed using sections stained with double immunofluorescence. Images were taken with a confocal microscope (LSM 510 Meta; Carl Zeiss) at $40 \times$ at a same optical slice thickness for all channels, and saved in TIFF format. The PV+, SP+ and CGRP+ axons that coexpress VGLUT2 in the peripheral and coronal pulp were counted from a total of 12-16 images from 3-4 sections of each of three human dental pulps. All axons longer than $1 \mathrm{~cm}$ in the images $(>45 \mu \mathrm{m}$ in tissue) were counted. Continuous strings of axonal beads with an apparent linear arrangement were considered to represent single axons. The differences among the three pulpal regions, and among the PV+, SP+ and CGRP+ axons was examined by one-way analysis of variance (ANOVA) and Scheffe's F-test (significance was set at $p<0.05$ ).

Electron microscopic immunohistochemistry. To improve penetration of antibodies for electron microscopy, sections were cryoprotected in 30\% sucrose in PBS overnight, frozen on dry ice for $30 \mathrm{~min}$, and thawed in PBS. They were then pretreated in PBS- buffered solutions of $1 \%$ sodium borohydride for $10 \mathrm{~min}, 3 \%$ $\mathrm{H}_{2} \mathrm{O}_{2}$ for $10 \mathrm{~min}$, and $10 \%$ NDS for $30 \mathrm{~min}$. After that, the sections were transferred to a solution of the primary antibody, mouse anti-PV at 1:1,000, rat anti-SP at 1:500, mouse anti-CGRP at 1:1,000, guinea pig anti-VGLUT2 at 1:500, mouse anti-syntaxin-I at 1:2,000 or rabbit anti-SNAP-25 at 1:3,000 for an overnight incubation at room temperature. After several rinses in PBS, sections were transferred to 2\% NDS for $30 \mathrm{~min}$ and then to an appropriate biotinylated secondary antibody, donkey anti-mouse, donkey anti-rat, donkey anti-guinea pig or donkey anti-rabbit at 1:200 for $2 \mathrm{~h}$. Avidin-biotin-peroxidase binding was with ExtrAvidin peroxidase (Sigma-Aldrich) at 1:5,000 for $1 \mathrm{~h}$, and peroxidase was revealed according to a standard diaminobenzidine (DAB) protocol. After additional staining in $1 \%$ osmium tetroxide in $\mathrm{PB}$ for $1 \mathrm{~h}$, the immunostained sections were dehydrated with a series of ethanol dilutions, and embedded in Durcupan ACM (Fluka, Buchs, Switzerland). The resin was cured 
in a $60^{\circ} \mathrm{C}$ oven for $48 \mathrm{~h}$. One square $\mathrm{mm}$ chips of the embedded tissue were cut out and glued onto support resin blocks with cyanoacrylate. Thin sections, cut with a diamond ultramicrotome knife (Dupont), were mounted in series on formvar-coated $\mathrm{Ni}$ grids and the grids were counterstained with uranyl acetate and lead citrate and examined on a Hitachi H-7500 electron microscope (Hitachi, Tokyo, Japan) at $80 \mathrm{kV}$. Photomicrographs were taken with a SC1000 CCD camera (Gatan, Pleasanton, CA) and saved as TIFF files using DigitalMicrograph software.

Immunohistochemical controls. All antibodies used in this study have been used in our laboratory and their specificity has been confirmed in previous experiments ${ }^{17,18,26,30}$. Nevertheless, we routinely processed sections where we omitted either the primary or the secondary antibodies. Sections incubated without the primary or secondary antibodies completely lacked specific staining. We also immunostained sections after preadsorption with blocking peptides, according to the respective supplier's protocol: for example, sections incubated with $18 \mu \mathrm{g} / \mathrm{mL}$ PV blocking peptide lacked specific immunostaining for PV. With electron microscopy, we studied the immunostaining in adjacent serial thin sections: consistent staining in serial sections of the same tissue elements confirmed specificity.

\section{Data availability}

The datasets generated and/or analyzed in the course the current study are available from the corresponding author on request.

Received: 5 February 2020; Accepted: 11 June 2020

Published online: 30 June 2020

\section{References}

1. Jackson, D. L., Aanonsen, L. M., Richardson, J. D., Geier, H. \& Hargreaves, K. M. An evaluation of the effects of excitatory amino acids in bovine dental pulp. Proc. Soc. Neurosci. 19, 996 (1993).

2. deGroot, J., Zhou, S. \& Carlton, S. M. Peripheral glutamate release in the hindpaw following low and high intensity sciatic stimulation. NeuroReport 11, 497-502. https://doi.org/10.1097/00001756-200002280-00014 (2000).

3. Du, J., Koltzenburg, M. \& Carlton, S. M. Glutamate-induced excitation and sensitization of nociceptors in rat glabrous skin. Pain 89, 187-198. https://doi.org/10.1016/s0304-3959(00)00362-6 (2001).

4. Lam, D. K., Sessle, B. J. \& Hu, J. W. Glutamate and capsaicin effects on trigeminal nociception I: activation and peripheral sensitization of deep craniofacial nociceptive afferents. Brain Res. 1251, 130-139. https://doi.org/10.1016/j.brainres.2008.11.029 (2009).

5. Paik, S. K. et al. Light and electron microscopic analysis of the somata and parent axons innervating the rat upper molar and lower incisor pulp. Neuroscience 162, 1279-1286. https://doi.org/10.1016/j.neuroscience.2009.05.046 (2009).

6. Narhi, M. \& Haegerstam, G. Intradental nerve activity induced by reduced pressure applied to exposed dentine in the cat. Acta Physiol. Scand. 119, 381-386. https://doi.org/10.1111/j.1748-1716.1983.tb07353.x (1983).

7. Narhi, M. V. Dentin sensitivity: a review. J. Biol. Buccale 13, 75-96 (1985).

8. Kim, Y. S. et al. Expression of metabotropic glutamate receptor mGluR5 in human dental pulp. J. Endod. 35, 690-694. https://doi. org/10.1016/j.joen.2009.02.005 (2009).

9. Paik, S. K. et al. Vesicular glutamate transporters in axons that innervate the human dental pulp. J. Endod. 38, 470-474. https:// doi.org/10.1016/j.joen.2011.12.012 (2012).

10. Yang, E. S. et al. Expression of vesicular glutamate transporters VGLUT1 and VGLUT2 in the rat dental pulp and trigeminal ganglion following inflammation. PLoS ONE 9, e109723. https://doi.org/10.1371/journal.pone.0109723 (2014).

11. Kim, Y. S., Kim, T. H., McKemy, D. D. \& Bae, Y. C. Expression of vesicular glutamate transporters in transient receptor potential melastatin 8 (TRPM8)-positive dental afferents in the mouse. Neuroscience 303, 378-388. https://doi.org/10.1016/j.neuroscien ce.2015.07.013 (2015).

12. Harris, R. \& Griffin, C. J. Fine structure of nerve endings in the human dental pulp. Arch. Oral Biol. 13, 773-778. https://doi. org/10.1016/0003-9969(68)90095-2 (1968).

13. Byers, M. R. Dental sensory receptors. Int. Rev. Neurobiol. 25, 39-94. https://doi.org/10.1016/s0074-7742(08)60677-7 (1984).

14. Norlin, T., Hilliges, M. \& Brodin, L. Immunohistochemical demonstration of exocytosis-regulating proteins within rat molar dentinal tubules. Arch. Oral Biol. 44, 223-231. https://doi.org/10.1016/s0003-9969(98)00115-0 (1999).

15. Honma, S., Taki, K., Lei, S., Niwa, H. \& Wakisaka, S. Immunohistochemical localization of SNARE proteins in dental pulp and periodontal ligament of the rat incisor. Anat. Rec. (Hoboken) 293, 1070-1080. https://doi.org/10.1002/ar.21106 (2010).

16. Honma, S., Kadono, K., Kawano, A. \& Wakisaka, S. Immunohistochemical localization of SNARE core proteins in intrapulpal and intradentinal nerve fibers of rat molar teeth. Arch. Oral Biol. 73, 248-252. https://doi.org/10.1016/j.archoralbio.2016.10.025 (2017).

17. Bae, J. Y., Kim, J. H., Cho, Y. S., Mah, W. \& Bae, Y. C. Quantitative analysis of afferents expressing substance P, calcitonin generelated peptide, isolectin B4, neurofilament 200, and Peripherin in the sensory root of the rat trigeminal ganglion. J. Comp. Neurol. 523, 126-138. https://doi.org/10.1002/cne.23672 (2015).

18. Bae, J. Y., Mun, C. J., Kim, Y. S., Ahn, D. K. \& Bae, Y. C. Quantitative ultrastructural analysis of fibers expressing parvalbumin, calretinin, calbindin D-28k, stage specific embryonic antigen-4, and phosphorylated neurofilament 200 in the peripheral sensory root of the rat trigeminal ganglion. J. Comp. Neurol. 526, 2204-2214. https://doi.org/10.1002/cne.24476 (2018).

19. Walsh, D. A., Mapp, P. I. \& Kelly, S. Calcitonin gene-related peptide in the joint: contributions to pain and inflammation. Br. J. Clin. Pharmacol. 80, 965-978. https://doi.org/10.1111/bcp.12669 (2015).

20. Bennett, M. R., Cheung, A. \& Brain, K. L. Sympathetic neuromuscular transmission at a varicosity in a syncytium. Microsc. Res. Tech. 42, 433-450. https://doi.org/10.1002/(SICI) 1097-0029(19980915)42:6<433::AID-JEMT6>3.0.CO;2-N (1998).

21. Burnstock, G. Non-synaptic transmission at autonomic neuroeffector junctions. Neurochem. Int. 52, 14-25. https://doi. org/10.1016/j.neuint.2007.03.007 (2008).

22. Jin, Y. H., Nishioka, H., Wakabayashi, K., Fujita, T. \& Yonehara, N. Effect of morphine on the release of excitatory amino acids in the rat hind instep: pain is modulated by the interaction between the peripheral opioid and glutamate systems. Neuroscience 138, 1329-1339. https://doi.org/10.1016/j.neuroscience.2005.12.049 (2006).

23. Svensson, P. et al. Glutamate-evoked pain and mechanical allodynia in the human masseter muscle. Pain 101, 221-227. https:// doi.org/10.1016/s0304-3959(02)00079-9 (2003).

24. Alstergren, P. et al. Glutamate-induced temporomandibular joint pain in healthy individuals is partially mediated by peripheral NMDA receptors. J. Orofac. Pain 24, 172-180 (2010). 
25. Cho, Y. S. et al. Rat odontoblasts may use glutamate to signal dentin injury. Neuroscience 335, 54-63. https://doi.org/10.1016/j. neuroscience.2016.08.029 (2016).

26. Kim, Y. S., Kim, S. K., Lee, J. S., Ko, S. J. \& Bae, Y. C. Expression of vesicular glutamate transporters in transient receptor potential ankyrin 1 (TRPA1)-positive neurons in the rat trigeminal ganglion. Brain Res. 1690, 31-39. https://doi.org/10.1016/j.brain res.2018.04.010 (2018).

27. Alvarez, F. J., Villalba, R. M., Zerda, R. \& Schneider, S. P. Vesicular glutamate transporters in the spinal cord, with special reference to sensory primary afferent synapses. J. Comp. Neurol. 472, 257-280. https://doi.org/10.1002/cne.20012 (2004).

28. Brumovsky, P., Watanabe, M. \& Hokfelt, T. Expression of the vesicular glutamate transporters-1 and -2 in adult mouse dorsal root ganglia and spinal cord and their regulation by nerve injury. Neuroscience 147, 469-490. https://doi.org/10.1016/j.neuroscien ce.2007.02.068 (2007).

29. Peng, Y. B., Ringkamp, M., Campbell, J. N. \& Meyer, R. A. Electrophysiological assessment of the cutaneous arborization of Adeltafiber nociceptors. J. Neurophysiol. 82, 1164-1177. https://doi.org/10.1152/jn.1999.82.3.1164 (1999).

30. Kim, T. H., Park, S. K., Choi, S. Y., Lee, J. S. \& Bae, Y. C. Morphologic change of parvalbumin-positive myelinated axons in the human dental pulp. J. Endod. 43, 977-981. https://doi.org/10.1016/j.joen.2017.01.010 (2017).

31. Medici, T. \& Shortland, P. J. Effects of peripheral nerve injury on parvalbumin expression in adult rat dorsal root ganglion neurons. BMC Neurosci. 16, 93. https://doi.org/10.1186/s12868-015-0232-9 (2015).

32. Sharma, N. et al. The emergence of transcriptional identity in somatosensory neurons. Nature 577, 392-398. https://doi. org/10.1038/s41586-019-1900-1 (2020).

33. Shigenaga, Y., Yoshida, A., Mitsuhiro, Y., Doe, K. \& Suemune, S. Morphology of single mesencephalic trigeminal neurons innervating periodontal ligament of the cat. Brain Res. 448, 331-338. https://doi.org/10.1016/0006-8993(88)91272-3 (1988).

34. Berman, L. H. \& Hartwell, G. R. Diagnosis. In Cohen's Pathway of the Pulp (eds Hargreaves, K. M. \& Cohen, S.) 2-39 (Elsevier/ Mosby, Amsterdam, 2011).

35. Pansecchi, D. Protection of the pulp. In Restorative Dentistry: Treatment Procedures and Future Prospects (ed. Brenna, F.) 137-160 (Elsevier/Mosby, Amsterdam, 2012).

36. Salih, V. \& Zaric, S. The periodontal ligament. In Essential Clinical Oral Biology (ed. Creanor, S.) 57-63 (Wiley-Blackwell, Hoboken, 2016).

37. Takashima, Y. et al. Diversity in the neural circuitry of cold sensing revealed by genetic axonal labeling of transient receptor potential melastatin 8 neurons. J. Neurosci. 27, 14147-14157. https://doi.org/10.1523/JNEUROSCI.4578-07.2007 (2007).

38. Lawson, S. N., McCarthy, P. W. \& Prabhakar, E. Electrophysiological properties of neurones with CGRP-like immunoreactivity in rat dorsal root ganglia. J. Comp. Neurol. 365, 355-366. https://doi.org/10.1002/(SICI)1096-9861(19960212)365:3<355::AIDCNE2 $>3.0 . C O ; 2-3$ (1996).

39. Nguyen, M. Q., Wu, Y., Bonilla, L. S., von Buchholtz, L. J. \& Ryba, N. J. P. Diversity amongst trigeminal neurons revealed by high throughput single cell sequencing. PLoS ONE 12, e0185543. https://doi.org/10.1371/journal.pone.0185543 (2017).

40. Byers, M. R. \& Cornel, L. M. Multiple complex somatosensory systems in mature rat molars defined by immunohistochemistry. Arch. Oral Biol. 85, 84-97. https://doi.org/10.1016/j.archoralbio.2017.09.007 (2018).

41. Landry, M., Bouali-Benazzouz, R., El Mestikawy, S., Ravassard, P. \& Nagy, F. Expression of vesicular glutamate transporters in rat lumbar spinal cord, with a note on dorsal root ganglia. J. Comp. Neurol. 468, 380-394. https://doi.org/10.1002/cne.10988 (2004).

42. Malet, M. \& Brumovsky, P. R. VGLUTs and glutamate synthesis-focus on DRG neurons and pain. Biomolecules 5, 3416-3437. https ://doi.org/10.3390/biom5043416 (2015).

43. Wada, H. et al. Elevated plasma levels of vascular endothelial cell markers in patients with hypercholesterolemia. Am. J. Hematol. 44, 112-116. https://doi.org/10.1002/ajh.2830440208 (1993).

\section{Acknowledgements}

The authors sincerely thank Dr. Juli Valtschanoff for helpful discussion and careful reading of the manuscript. This work was supported by the National Research Foundation of Korea (NRF) grant funded by the Korea government (MSIT, NRF-2017R1A5A2015391, NRF-2017R1A2B2003561).

\section{Author contributions}

All authors have full access to all the data in this study and take responsibility for the integrity of the data and the accuracy of the data analysis. S.Y.C., J.W.K. and Y.C.B. designed the study. S.K.P., S.K.C., Y.G.K., S.H.S. and J.H.L. performed immunohitochemistry experiments and electron microscopy. S.K.P., S.K.C. and Y.C.B. analyzed and interpreted the data. All authors participated in the writing of the manuscript and approved the final version of the manuscript.

\section{Competing interests}

The authors declare no competing interests.

\section{Additional information}

Correspondence and requests for materials should be addressed to Y.C.B.

Reprints and permissions information is available at www.nature.com/reprints.

Publisher's note Springer Nature remains neutral with regard to jurisdictional claims in published maps and institutional affiliations.

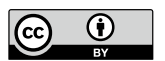

Open Access This article is licensed under a Creative Commons Attribution 4.0 International License, which permits use, sharing, adaptation, distribution and reproduction in any medium or format, as long as you give appropriate credit to the original author(s) and the source, provide a link to the Creative Commons license, and indicate if changes were made. The images or other third party material in this article are included in the article's Creative Commons license, unless indicated otherwise in a credit line to the material. If material is not included in the article's Creative Commons license and your intended use is not permitted by statutory regulation or exceeds the permitted use, you will need to obtain permission directly from the copyright holder. To view a copy of this license, visit http://creativecommons.org/licenses/by/4.0/.

(c) The Author(s) 2020 